\title{
Physiological hyperinsulinaemia increases intramuscular microvascular reactive hyperaemia and vasomotion in healthy volunteers
}

\author{
R. T. de Jongh ${ }^{1}$ - A. D. H. Clark ${ }^{2}$ R. G. IJzerman ${ }^{1}$ E. H. Serné ${ }^{~}$ G. de Vries ${ }^{1}$ C. D. A. Stehouwer ${ }^{1}$ \\ ${ }^{1}$ Department of Internal Medicine and Institute for Cardiovascular Research, VU University Medical Center, Amsterdam, \\ The Netherlands \\ ${ }^{2}$ Division of Biochemistry, Medical School, University of Tasmania, Hobart, Australia
}

\section{Abstract}

Aims/hypothesis. Insulin possesses vasodilatory actions that may be important in regulating its access to insulin-sensitive tissues. Our study aims to directly measure changes in response to insulin in the human skeletal muscle microcirculation. Measurement was by an implanted laser Doppler probe.

Methods. We investigated changes in intramuscular and skin microvascular perfusion in 12 healthy individuals during a hyperinsulinaemic and a control clamp. We determined leg blood flow with plethysmography, finger skin functional capillary recruitment with capillaroscopy, endothelium-(in)dependent vasodilation by iontophoresis of acetylcholine and sodium nitroprusside, and leg intramuscular reactive hyperaemia and vasomotion with laser Doppler measurements.

Results. Compared to the control study, hyperinsulinaemia $(416 \pm 82 \mathrm{pmol} / \mathrm{l})$ caused: (i) an increase in leg blood flow $\left(1.0 \pm 1.0\right.$ vs $0.1 \pm 0.6 \mathrm{ml} \cdot \mathrm{min}^{-1} .100 \mathrm{ml}$, $p<0.05)$; (ii) an increase in finger skin capillary recruitment (14.9 \pm 10.1 vs $-5.6 \pm 11.0 \%, p<0.01)$; (iii) no change in baseline laser Doppler perfusion either in finger skin or leg muscle; (iv) a tendency to increase acetylcholine-mediated vasodilation $(475 \pm 534$ vs $114 \pm 337 \%, p=0.07)$ with no change in sodiumnitroprusside-mediated vasodilation $(p=0.2)$ in finger skin; (v) an increase in intramuscular reactive hyperaemia $(423 \pm 507$ vs $0 \pm 220 \%, p<0.01)$; and (vi) a decrease in time needed to reach peak intramuscular perfusion $(-3.6 \pm 3.0$ vs $1.1 \pm 3.1 \mathrm{~s}, p<0.01)$. In addition, hyperinsulinaemia induced an increase in intramuscular vasomotion by increasing the contribution of frequencies between 0.01 and $0.04 \mathrm{~Hz}(p<0.05$ for all), which probably represents increased endothelial and neurogenic activity.

Conclusions/interpretation. Physiological hyperinsulinaemia not only stimulates total blood flow and skin microvascular perfusion, but also augments human skeletal muscle microvascular recruitment and vasomotion as detected directly by laser Doppler measurements.

Keywords Insulin - Laser Doppler flow · Microcirculation $\cdot$ Muscle $\cdot$ Reactive hyperaemia Skin · Vasomotion
Received: 23 December 2003 / Accepted: 1 March 2004 Published online: 28 May 2004

(C) Springer-Verlag 2004

C. D. A. Stehouwer $(\bullet)$

Department of Internal Medicine

and Institute for Cardiovascular Research,

VU University Medical Center, De Boelelaan 1117,

PO Box 7057, 1007 MB Amsterdam, The Netherlands

E-mail: cda.stehouwer@vumc.nl

Tel.: +31-20-4440629, Fax: +31-20-4444313

Abbreviations: Ach, acetylcholine $\cdot$ NO, nitric oxide . PU, arbitrary perfusion units $\cdot$ SNP, sodium nitroprusside

\section{Introduction}

Skeletal muscle is the main peripheral site of insulinmediated glucose uptake and vasodilation [1]. Defects in this haemodynamic effect of insulin may contribute to diminished insulin-mediated glucose uptake and therefore play an important pathophysiological role in insulin resistance in Type 2 diabetes, obesity and hypertension [1].

In previous studies, insulin has been shown to modulate microvascular perfusion. In the rat hindlimb, insulin recruited capillary perfusion as shown by an in- 


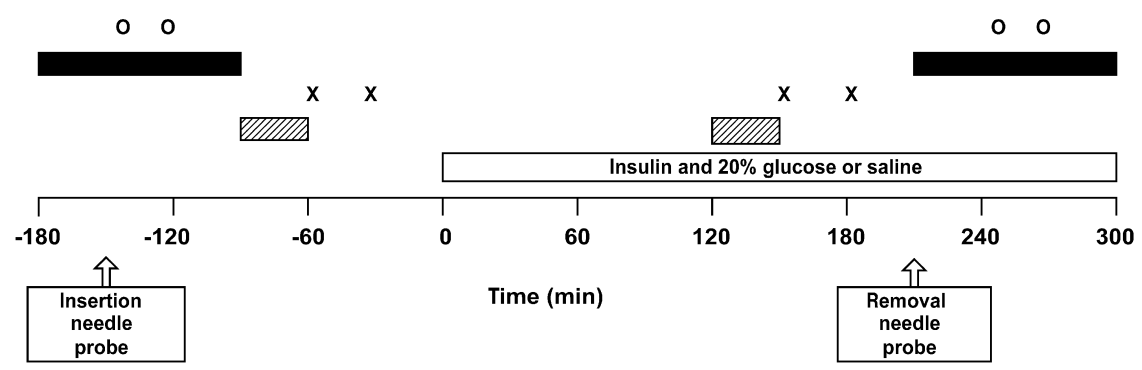

Fig. 1. Design of the study protocol. O: leg blood flow and blood pressure measurements; X: intramuscular peak reactive hyperaemia; hatched rectangle: intramuscular vasomotion; black rectangle: capillaroscopy and endothelium-(in)dependent vasodilation in skin of the finger

crease in the metabolism of 1-methylxanthine. This capillary recruitment was related to changes in skeletal muscle glucose uptake independently of changes in total blood flow [2, 3]. Furthermore, it has been shown that laser Doppler flow measurements in the constant-flow, erythrocyte-perfused rat hindlimb correlate with changes in muscle metabolism, indicating the ability of this technology to measure erythrocyte movement both proportional to nutritive flow and separate from total flow [4]. In rat muscle in vivo, physiological hyperinsulinaemia induced an increase in the laser Doppler signal that is consistent with nutritive flow recruitment without a change in total flow [5].

In humans, hyperinsulinaemia increased the distribution volume of glucose [6] and specifically increased capillary blood volume as measured with contrast-enhanced ultrasound [7]. In human skin, we have recently shown that insulin is capable of inducing functional recruitment of capillaries [8]. In addition, insulin influenced rhythmic fluctuations of the skin microvasculature (so-called vasomotion) by increasing the relative contribution of endothelial activity. This implies that insulin modulates microcirculatory flow through a mechanism that is dependent on the endothelium [8]. Although the above-mentioned studies are consistent with the concept of an insulinmediated effect on intramuscular microvascular perfusion, such an effect has never been directly assessed in humans by means of intramuscular laser Doppler measurements.

In human skin, reactive hyperaemia after arterial occlusion as measured with laser Doppler is commonly applied to assess microvascular vasodilatory capacity $[9,10]$. The same technique can also be applied to the vasculature of human skeletal muscle. If the probe position remains unchanged, the reproducibility of intramuscular reactive hyperaemia measurements after arterial occlusion has been shown to be excellent [11, 12].

The aim of the present study was to use an implanted laser Doppler probe to examine directly in human
Table 1. Characteristics of the study population

\begin{tabular}{ll}
\hline Characteristic & $\begin{array}{l}\text { Mean } \pm \text { SD or } \\
\text { number }\end{array}$ \\
\hline Number (men/women) & $12(6 / 6)$ \\
Age (years) & $27 \pm 8$ \\
BMI $\left(\mathrm{kg} / \mathrm{m}^{2}\right)$ & $22.6 \pm 1.0$ \\
WHR & $0.81 \pm 0.05$ \\
M/I-value $\left(\mathrm{mg} \cdot \mathrm{kg}^{-1} \cdot \mathrm{min}^{-1}\right.$ per pmol/l) $* 100$ & $2.0 \pm 0.6$ \\
Total cholesterol $\left(\mathrm{mmol}^{\prime} \mathrm{l}\right)$ & $4.2 \pm 1.0$ \\
LDL-cholesterol $(\mathrm{mmol} / \mathrm{l})$ & $2.3 \pm 0.9$ \\
HDL-cholesterol $(\mathrm{mmol} / \mathrm{l})$ & $1.4 \pm 0.3$ \\
Triglycerides $(\mathrm{mmol} / \mathrm{l})$ & $0.9 \pm 0.5$ \\
\hline
\end{tabular}

M/I-value $=$ glucose infusion rate during the hyperinsulinaemic clamp, expressed per kg body weight, corrected for insulin concentrations in pmol per litre

muscle the insulin-mediated effects (i) on microvascular reactive hyperaemia after arterial occlusion and (ii) on microvascular vasomotion, and to compare these effects with insulin-mediated effects on total blood flow and skin microvascular perfusion.

\section{Subjects and methods}

Subjects. Characteristics of the study population are shown in Table 1. Participants were healthy as judged by medical history. They were also non-diabetic [13], normotensive $(<140 /<90 \mathrm{~mm} \mathrm{Hg})$ as determined by triplicate office blood pressure measurement, non-smokers and not taking any medication except oral contraceptives. All participants gave informed consent for participation in the study. The study was undertaken with approval of the local ethics committee and performed in accordance with the Declaration of Helsinki.

Study design. All individuals underwent the protocol as described in Figure 1. All measurements were performed in a quiet, temperature-controlled room $\left(23.4 \pm 0.5^{\circ} \mathrm{C}\right)$ and after a 10-hour fast.

Throughout the study, laser Doppler measurements were performed in the anterior tibial muscle. The laser Doppler signal is determined by the product of local speed and concentration of moving blood cells [14]. The exact volume measured by laser Doppler cannot be determined, and consequently, the laser Doppler signal is expressed in arbitrary units. Each time the laser Doppler probe is applied to a new volume of tissue the optical properties and microvascular structure of the tissue being monitored will be different. However, so long as these immeasurable variables remain constant, the laser Doppler signal is very useful because any changes that are seen are strongly related to changes occurring in local perfusion [14]. 
Thus, only responses to provocational manoeuvres and not the absolute laser Doppler signal can be compared between individuals [14].

Laser Doppler perfusion in the anterior tibial muscle was measured with a needle probe (model 402, fibre separation $=0.15 \mathrm{~mm}$; Perimed, Stockholm, Sweden) sterilised in ethylene oxide. A standard cannula (Venflon $0.9 \times 25 \mathrm{~mm}$; B-D) was inserted into the anterior tibial muscle approximately $20 \mathrm{~cm}$ proximal to the ankle joint of the non-dominant leg. The needle probe was introduced through the cannula, positioned to protrude $0.5 \mathrm{~cm}$ from the tip of the cannula in order to make proper contact with the muscle tissue, as indicated by a regular and pulsatile laser Doppler signal, and fixated on the skin with a special fixation device (PH 18 Fixation Device; Perimed). During the studies subjects were in a supine position. In order to minimise movement artefacts, the leg was fixated by resting on a vacuum cushion moulded to hold the limb in a comfortable position.

Blood pressure. Blood pressure measurements were performed as depicted in Figure 1 (Colin Press-Mate BP-8800; Colin, Komaki City, Japan). The average of three consecutive blood pressure readings during each period was used for further analyses.

Hyperinsulinaemic-euglycaemic clamp. Insulin sensitivity was determined with the euglycaemic-hyperinsulinaemic clamp method as described previously [15]. Insulin (Velosulin; Novo Nordisk, Bagsvaerd, Denmark) was infused at a rate of $50 \mathrm{mU} \cdot \mathrm{kg}^{-1} \cdot \mathrm{h}^{-1}$.

To exclude non-specific changes in vascular measurements, a time- and volume-control study was performed in an identical fashion. We used hypotonic saline $(0.65 \%)$ as control infusion, because glucose infusion will behave like a hypotonic fluid and will increase both the intra- and extracellular fluid volume.

Leg blood flow. Leg blood flow was measured by venous occlusion plethysmography (EC6; Hokanson, Bellevue, Wash., USA) in a supine position with the foot elevated [8]. An occlusive cuff was placed proximally around the dominant leg and the strain gauge was placed around the calf at the largest circumference. Hokanson dedicated software was used (NIVP3; Hokanson) to inflate the cuff to $50 \mathrm{~mm} \mathrm{Hg}$, capture the inflow waveform and calculate blood flow. A paediatric cuff inflated to suprasystolic pressure was placed around the ankle in order to exclude the foot circulation. Leg blood flow measurements represent the average of seven to nine recordings. The mean of two consecutive measurements was used for further analyses. The day-to-day coefficient of variation was $9.6 \pm 7.4 \%$, as determined in ten healthy individuals on two separate days.

Capillaroscopy and endothelium-(in)dependent vasodilation in skin of the finger. Nailfold capillary studies and iontophoresis studies were performed as described previously [8]. Perfused capillary density was defined as the number of erythrocyteperfused capillaries per square millimetre. Reactive hyperaemia after 4 minutes of arterial occlusion was used to assess functional capillary recruitment. This was calculated by dividing the increase in perfused capillary density during reactive hyperaemia by the baseline perfused capillary density. The day-to-day coefficient of variation of functional capillary recruitment was $15.9 \pm 8.0 \%$ as determined in ten healthy individuals on two separate days.

Microvascular endothelium-(in)dependent vasodilation was determined as described previously [8]. Acetylcholine (1\% Miochol, IOLAB, Bourneville Pharma, Braine l'Alleud, Bel- gium) was delivered on the middle phalanx of the third finger of the non-dominant hand using an anodal current. Delivery consisted of seven doses $(0.1 \mathrm{~mA}$ for $20 \mathrm{~s})$ with a 60 -s interval between each dose. Sodium nitroprusside $(0.01 \%$, Nipride; Roche, Almere, The Netherlands) was delivered on the same spot of the fourth finger using a cathodal current, consisting of nine doses $(0.2 \mathrm{~mA}$ for $20 \mathrm{~s})$ with a 90 -s interval between each dose. The day-to-day coefficient of variation of the relative increase from baseline to the final two deliveries was $12.2 \pm 9.7 \%$ for acetylcholine and $16.4 \pm 8.1 \%$ for sodium nitroprusside, as determined in ten healthy individuals on two separate days. Skin temperature was above $30{ }^{\circ} \mathrm{C}$ during all measurements.

Reactive hyperaemia in muscle of the lower leg. Intramuscular reactive hyperaemia measurements were started at least 45 minutes after muscle puncture and after stable laser Doppler recordings had been achieved. An occlusive cuff was placed around the distal thigh of the non-dominant leg. After 5 minutes of resting laser Doppler recording, the cuff was rapidly inflated to $200 \mathrm{~mm} \mathrm{Hg}$. This pressure was maintained for 3 minutes, which was shown by others [11] to give a maximal hyperaemic response. After release of the cuff, intramuscular reactive hyperaemia was recorded. Baseline perfusion was calculated as the mean value of the 3-minute laser Doppler recording immediately prior to inflation of the cuff. The peak perfusion corresponded with the maximal laser Doppler signal recorded after release of the cuff. The time to peak perfusion was the time between the start of cuff deflation and the peak perfusion. Reactive hyperaemia was measured twice with a 20 -minute interval. The mean of these two measurements was used in further analyses. For two consecutive measurements in 12 healthy subjects, the coefficient of variation of the relative increase in perfusion and the time needed to reach peak perfusion during reactive hyperaemia were $10.2 \pm 4.3 \%$ and $16.2 \pm 11.4 \%$ respectively.

Vasomotion in the muscle of the lower leg. The laser Doppler signal was recorded for 30 minutes and vasomotion analyses were performed on a continuous 15-minute sample relatively free of movement artefacts. A bandpass filter, with cut-off frequencies at $20 \mathrm{~Hz}$ and $20 \mathrm{kHz}$ and a time-constant of $0.2 \mathrm{~s}$, was used. We used Fourier transformation to determine the contribution of different frequency components to the variability of the laser Doppler signal. The frequency band between 0.01 and 1.6 Hz was studied and divided into a set of intervals. Based upon the work of Stefanovska et al. [16], we chose five frequency intervals: (i) $0.01-0.02 \mathrm{~Hz}$, which is thought to contain local endothelial activity; (ii) $0.02-0.06 \mathrm{~Hz}$, which is thought to contain neurogenic activity; (iii) $0.06-0.15 \mathrm{~Hz}$, which is associated with the myogenic response of the smooth muscle cells in the vessel wall; (iv) $0.15-0.4 \mathrm{~Hz}$, which is the frequency interval of respiratory function; and (v) $0.4-1.6 \mathrm{~Hz}$, which contains the heart beat frequency. Data were processed on a personal computer using Perisoft dedicated software (PSW version 3.11, Perimed). The day-to-day coefficients of variation of the energy density of the frequency intervals $0.01-0.02$, $0.02-0.06,0.06-0.15,0.15-0.40$ and $0.40-1.6 \mathrm{~Hz}$ were $23.1 \pm 19.0 \%, 26.8 \pm 25.4 \%, 25.5 \pm 22.7 \%, 27.9 \pm 22.5 \%$ and $27.5 \pm 17.5 \%$ respectively in 12 healthy subjects.

Analytical methods. Plasma insulin concentrations were measured by radioimmunoassay techniques (Medgenix Diagnostics, Fleurus, Belgium). Blood glucose concentrations were determined by the glucose oxidase method with a glucose analyser YSI2300 (Yellow Springs Instrument, Yellow Springs, Ohio, USA). Fasting serum total cholesterol, HDL cholesterol and triglyceride concentrations were measured by enzymatic 
Table 2. Metabolic and haemodynamic variables before and during the hyperinsulinaemic clamp and time-volume control study

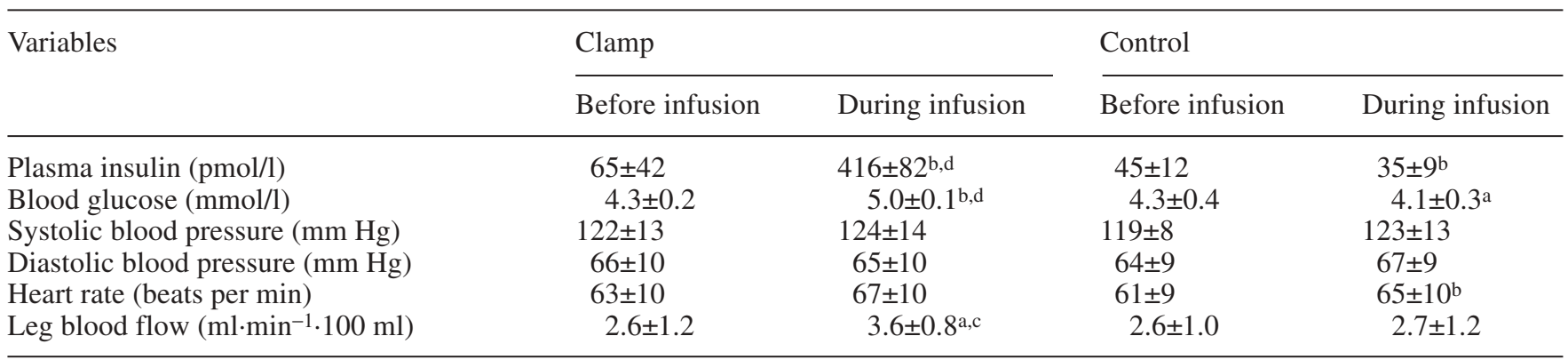

${ }^{\mathrm{a}} p<0.05$ and ${ }^{\mathrm{b}} p<0.01$ during vs before infusion; ${ }^{\mathrm{c}} p<0.05$ and ${ }^{\mathrm{d}} p<0.01$ for change during insulin versus during control study

Table 3. Skin microcirculatory measurements before and during the hyperinsulinaemic clamp and time-volume control study

\begin{tabular}{|c|c|c|c|c|}
\hline & \multicolumn{2}{|l|}{ Clamp } & \multicolumn{2}{|l|}{ Control } \\
\hline & Before infusion & During infusion & Before infusion & During infusion \\
\hline Baseline capillary density (no./mm²) & $33.8 \pm 5.5$ & $33.8 \pm 5.8$ & $34.6 \pm 5.6$ & $36.6 \pm 6.6$ \\
\hline Peak capillary density (no./mm²) & $49.0 \pm 8.0$ & $53.3 \pm 10.0^{\mathrm{a}, \mathrm{d}}$ & $50.5 \pm 9.2$ & $50.9 \pm 8.0$ \\
\hline \multicolumn{5}{|l|}{ Ach-mediated vasodilation } \\
\hline Baseline laser Doppler perfusion (PU) & $24.0 \pm 9.7$ & $20.5 \pm 7.5$ & $28.8 \pm 8.5$ & $26.4 \pm 12.0$ \\
\hline Plateau laser Doppler perfusion (PU) & $148.7 \pm 79.3$ & $204.8 \pm 78.0^{\mathrm{b}}$ & $197.0 \pm 124.7$ & $191.8 \pm 70.0$ \\
\hline Ach-mediated vasodilation $(\%)$ & $491 \pm 196$ & $1000 \pm 499 \mathrm{~b}, \mathrm{c}$ & $593 \pm 355$ & $707 \pm 268$ \\
\hline \multicolumn{5}{|l|}{ SNP-mediated vasodilation } \\
\hline
\end{tabular}

Ach, acetylcholine; no, number of perfused capillaries; SNP, sodium nitroprusside; PU, arbitrary perfusion units. a $p<0.05$ and ${ }^{\mathrm{b}} p<0.01$ during vs before infusion; ${ }^{\mathrm{c}} p=0.07,{ }^{\mathrm{d}} p<0.05$ and ${ }^{\mathrm{e}} p<0.01$ for change during insulin versus during control study

techniques (Hitachi 747 model 100; Roche diagnostics, Mannheim, Germany). Fasting LDL cholesterol was calculated by the Friedewald formula.

Statistical analyses. All variables were tested for normality of distribution. Data are expressed as means \pm SD or median (interquartile range) as appropriate. The paired Student's $t$ test and the Wilcoxon signed rank test for paired data were used to study effects of the hyperinsulinaemic clamp and the control study, and to compare these effects. Pearson's correlation analysis was used to study associations between insulin-mediated effects on total blood flow and intramuscular and skin microvascular perfusion. A two-tailed $p$ value of less than 0.05 was considered significant. All analyses were performed on a personal computer using the statistical software package SPSS version 11.0.

\section{Results}

Metabolic and haemodynamic variables before and during the hyperinsulinaemic clamp. The change in glucose concentration during the hyperinsulinaemic- euglycaemic clamp was significantly different from that in the control study $(0.6 \pm 0.3 \mathrm{vs}-0.2 \pm 0.2 \mathrm{mmol} / \mathrm{l}$, $p<0.01$; Table 2), because the plasma glucose concentration was clamped at $5 \mathrm{mmol} / \mathrm{l}$. During the clamp, insulin levels increased from $65 \pm 42 \mathrm{pmol} / \mathrm{l}$ to $416 \pm 82 \mathrm{pmol} / \mathrm{l}$. In comparison with the control study, hyperinsulinaemia increased leg blood flow $(1.0 \pm 1.0$ vs $0.1 \pm 0.6 \mathrm{ml} \cdot \mathrm{min}^{-1} \cdot 100 \mathrm{ml}, p<0.05$ ).

Capillaroscopy and endothelium-(in)dependent vasodilation in skin of the finger. As compared with the control study, hyperinsulinaemia did not influence baseline perfused capillary density, but did increase functional capillary recruitment during peak reactive hyperaemia $(14.9 \pm 10.1$ vs $-5.6 \pm 11.0 \%$, $p<0.01$; Table 3). Hyperinsulinaemia also tended to increase acetylcholine-mediated vasodilation in comparison with the control study $(475 \pm 534$ vs $114 \pm 337 \%$-points, $p=0.07$ ), but did not significantly change vasodilation mediated by sodium-nitroprusside $(p=0.2)$. 
Table 4. Reactive hyperaemia measurements in the tibial muscle of the lower leg before and during the hyperinsulinaemic clamp and time-volume control study

\begin{tabular}{|c|c|c|c|c|}
\hline & \multicolumn{2}{|l|}{ Clamp } & \multicolumn{2}{|l|}{ Control } \\
\hline & Before infusion & During infusion & Before infusion & During infusion \\
\hline Peak perfusion (PU) & $193.7 \pm 100.0$ & $411.6 \pm 203.0^{b, c}$ & $176.2 \pm 69.6$ & $181.5 \pm 83.5$ \\
\hline Absolute increase (PU) & $158.0 \pm 97.8$ & $368.8 \pm 202.4^{\mathrm{a}, \mathrm{c}}$ & $146.3 \pm 68.4$ & $153.3 \pm 76.1$ \\
\hline Relative increase (\%) & $565 \pm 358$ & $987 \pm 605^{\mathrm{a}, \mathrm{c}}$ & $622 \pm 337$ & $633 \pm 366$ \\
\hline
\end{tabular}

PU, arbitrary perfusion units; a $p<0.05,{ }^{\mathrm{b}} p<0.01$ during vs before infusion; ${ }^{\mathrm{c}} p<0.01$ for change during insulin versus during control study

Table 5. Energy densities of microvascular laser Doppler perfusion within each of the five frequency intervals and the total energy density in muscle of the lower leg before and during the hyperinsulinaemic clamp and time-volume control study

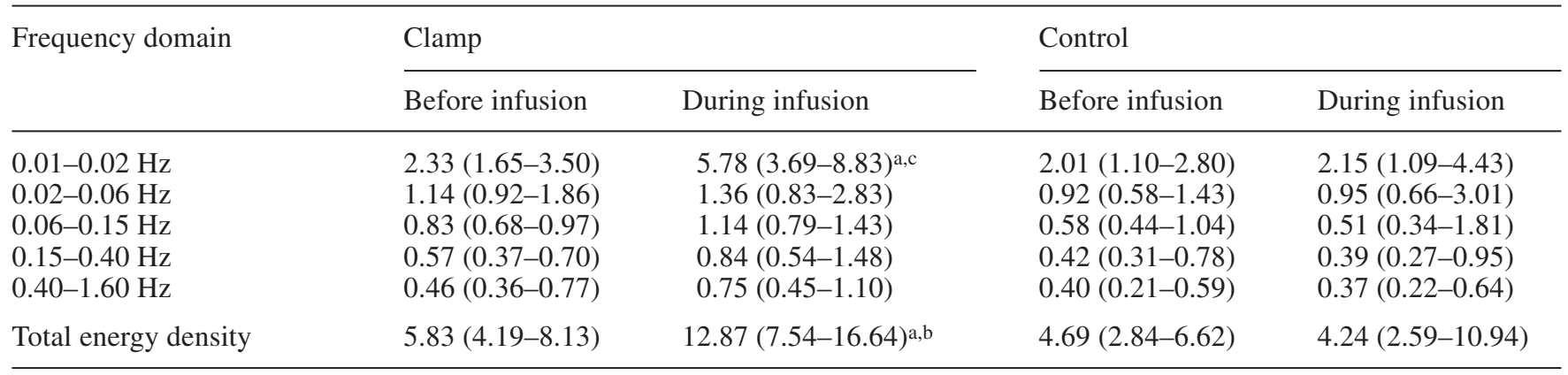

Values are expressed as median (interquartile range); ${ }^{\mathrm{a}} p<0.01$ during vs before infusion; ${ }^{\mathrm{b}} p<0.05$ and ${ }^{\mathrm{c}} p<0.01$ for change during insulin study versus during control study

Similar results were obtained if the absolute increase in perfused capillary density and in laser Doppler perfusion during iontophoresis of acetylcholine and sodium nitroprusside was used instead of the percentage increase (data not shown).

Reactive hyperaemia in muscle of the lower leg. Compared to the control study, insulin did not influence basal laser Doppler perfusion $(p=0.3)$. However, hyperinsulinaemia increased peak laser Doppler perfusion (by $218.0 \pm 166.3$ vs $5.3 \pm 97.1 \mathrm{PU}, p<0.01$; Table 4) and also raised the absolute and relative increases in laser Doppler perfusion after arterial occlusion (by $210.8 \pm 155.9$ vs $7.1 \pm 74.3 \mathrm{PU}$, and by $414 \pm 327$ vs $0 \pm 220 \%$-points respectively, $p<0.01$ for both; Table 4 , Fig. 2). In addition, hyperinsulinaemia decreased the time needed to reach peak laser Doppler perfusion after arterial occlusion $(-3.7 \pm 3.1$ vs $1.1 \pm 3.1 \mathrm{~s}, p<0.01)$. Pearson's correlation analysis demonstrated that insulin-mediated changes in absolute and relative increase after arterial occlusion and time needed to reach peak laser Doppler perfusion were not significantly associated with insulin-mediated effects on total leg blood flow (Pearson's correlation coefficient; $r=0.19, p=0.5$; $r=0.15, p=0.6$ and $r=-0.16, p=0.5$, respectively).

Vasomotion in the muscle of the lower leg. Table 5 shows energy densities of the intramuscular laser

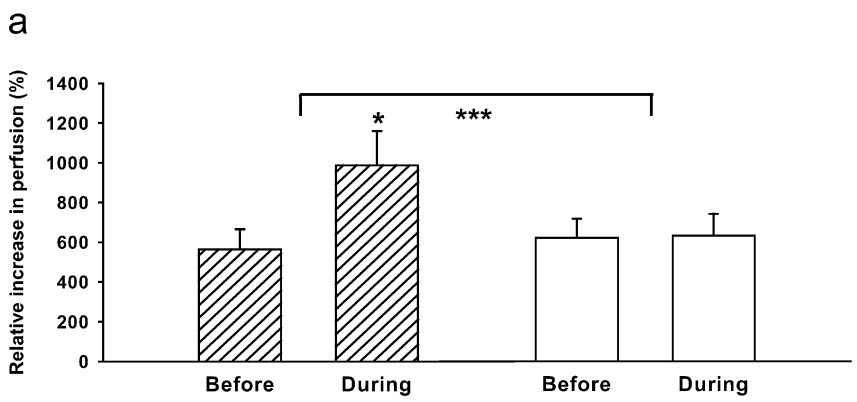

b

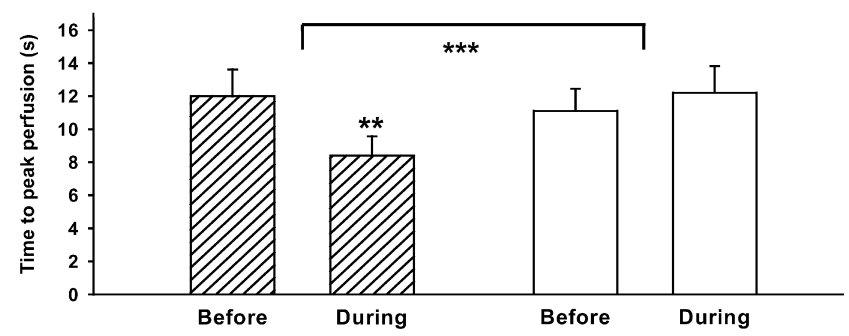

Fig. 2. The relative increase in intramuscular perfusion after arterial occlusion (a) and time needed to reach peak intramuscular perfusion (b). Both were measured before and during the hyperinsulinaemic clamp (hatched bars) and the control study (white bars). ${ }^{*} p<0.05$ and ${ }^{* *} p<0.01$ during vs before infusion; ${ }^{* * *} p<0.01$ for change during insulin versus during control study 
a

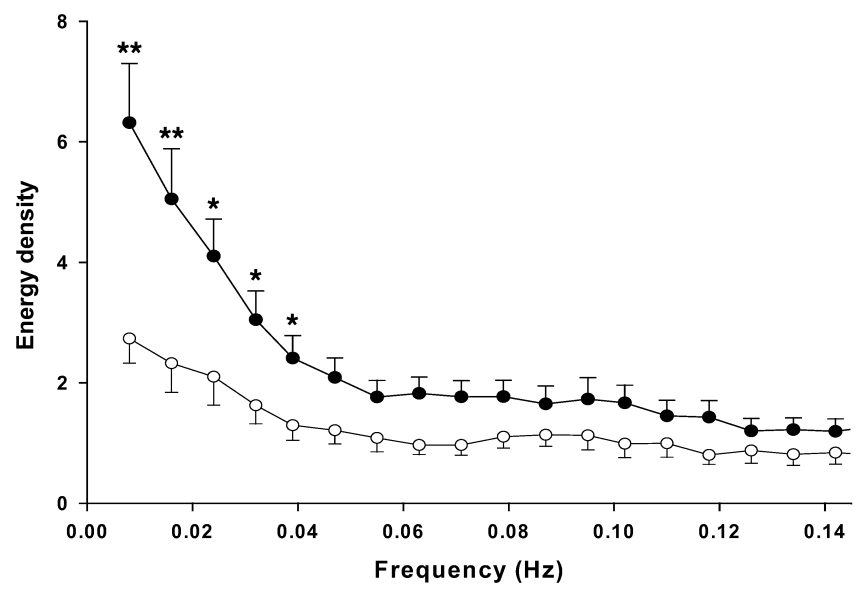

b

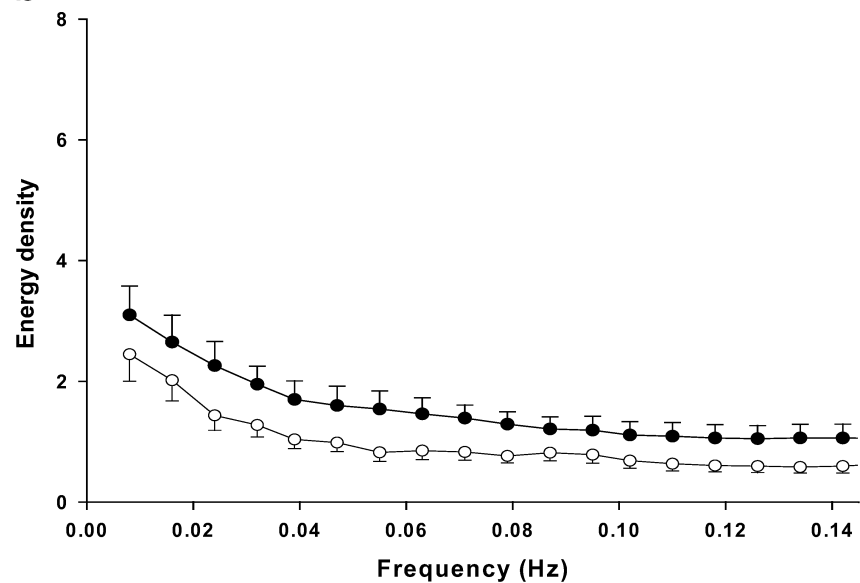

Fig. 3. The contribution of frequencies between 0 and $0.15 \mathrm{~Hz}$ to the total energy density of microvascular intramuscular vasomotion before (white circles) and during (black circles) the hyperinsulinaemic clamp (a) and the control study (b). ${ }^{*} p<0.05$ and ${ }^{* *} p<0.01$ for change during insulin versus during control study

Doppler signal within each frequency interval and the calculated total energy density within the interval from 0.01 to $1.6 \mathrm{~Hz}$. Compared with the control study, hyperinsulinaemia increased the intramuscular energy density of the frequency intervals between 0.01 and $0.02 \mathrm{~Hz}$ by (median value and interquartile range): $3.37(1.56-4.2)$ vs 0.44 ( -1.02 to 2.28$), p<0.01$. It increased the calculated total energy density by: 5.88 (1.46-8.38) vs $0.46(-1.59$ to 5.32$), p<0.05$. To illustrate the effect of hyperinsulinaemia at the lower frequencies of the frequency interval between 0.01 and $1.6 \mathrm{~Hz}$, its effect on the energy densities of each of the registered frequencies between 0.01 and $0.15 \mathrm{~Hz}$ was measured (Fig. 3). Compared with the control group, hyperinsulinaemia increased the contribution of all frequencies registered between 0.01 and $0.04 \mathrm{~Hz}$ in the muscle, but not of the frequencies above $0.04 \mathrm{~Hz}$.

\section{Discussion}

The main finding of this study is that insulin affects intramuscular microvascular perfusion as estimated with the laser Doppler technique. We show that hyperinsulinaemia increases two different measures of intramuscular microvascular activity: reactive hyperaemia after arterial occlusion and vasomotion. Hyperinsulinaemia increases the maximal reactive response in intramuscular laser Doppler perfusion following 3 minutes of arterial occlusion and reduces the time needed to reach this maximal response. In addition, hyperinsulinaemia increases intramuscular microvascular vasomotion, in a way that indicates an increased contribution of endothelial and neurogenic activity. We also confirmed previous findings of an insulin-induced increase in total leg blood flow, in skin capillary recruitment after arterial occlusion, and in endotheliumdependent vasodilation $[8,17]$. Taken together, these data show that the vascular effects of insulin are not limited to total blood flow and skin microvascular function during provocational manoeuvres, but extend to microvascular perfusion in human muscle.

The present results are in agreement with previous studies that have demonstrated insulin-mediated effects on microvascular perfusion in human muscle using positron emission tomography or contrastenhanced ultrasound $[6,7]$. However, these methods do not measure muscle perfusion in real time and are therefore not able to measure vasomotion.

In connection with our finding that hyperinsulinaemia stimulated the response of intramuscular microvascular perfusion during reactive hyperaemia, it is possible that the stimulatory effect of hyperinsulinaemia is due to a decreased sensitivity to vasoconstrictor or an increased sensitivity to vasodilatory stimuli. The mechanisms responsible for the increase in vascular perfusion after arterial occlusion are not fully understood. The accumulation of metabolic vasodilators, myogenic and/or neurogenic mechanisms may play a role [18]. It seems unlikely that hyperinsulinaemia decreases sensitivity to vasoconstrictive effects, because, both in the isolated rat arteriole and in the human forearm, hyperinsulinaemia has been shown to increase vasoconstrictive effects by inducing endothelin-1 activity $[19,20]$. In humans, moreover, insulin has been shown to stimulate vasodilatory effects by inducing endothelial nitric oxide (NO) production [21]. The idea that NO plays a part in intramuscular reactive hyperaemia is supported by most $[22,23,24]$, but not all studies [25] using venous occlusion plethysmography. Therefore, in the present study, enhanced endothelial NO production might have contributed to insulin-mediated changes in intramuscular reactive hyperaemia. Evidently this and other possible mechanisms in intramuscular reactive hyperaemia, as well as the insulin-mediated effects on these mechanisms, need to be further investigated. 
In our study, as in many previous studies, insulin increased total leg blood flow $[8,17,26]$. This may have contributed to insulin-mediated changes in intramuscular reactive hyperaemia. However, insulin-mediated changes in intramuscular reactive hyperaemia were not associated with insulin-mediated changes in total leg blood flow. In addition, iontophorised insulin can directly increase skin microvascular perfusion, indicating a direct local microvascular effect of insulin [8]. Moreover, animal and human studies suggest that insulin-induced changes in basal microvascular perfusion occur before changes in total blood flow demonstrating an independency of both phenomena $[5,7,27]$.

The present study demonstrated insulin-mediated differences in capillary density and microvascular perfusion after provocational manoeuvres, i.e. arterial occlusion or acetylcholine administration, but not in the basal state. This is in contrast with previous studies $[5,8]$ and may be explained by a lower sensitivity of the methods presently used and by the small number of participants. It should be emphasised that our study was not specifically designed, and thus was underpowered, to examine the effects of insulin on baseline microvascular perfusion, and such effects therefore cannot be excluded.

Intramuscular reactive hyperaemia measurements with the laser Doppler have been used and validated in previous studies $[11,12,28]$. Calculation of the relative increase in intramuscular perfusion allows correction for differences in baseline intramuscular perfusion. This is important, because previous studies have shown that intramuscular laser Doppler perfusion at rest shows considerable spatial variation at different measurement sites [11, 12]. Our experiments were performed in conscious, healthy subjects and so slight changes in the probe measurement angle and depth could not be avoided. The laser Doppler signal consists of the average perfusion in all arterioles, venules and capillaries of a certain measured volume of muscle tissue. Slight changes in this measured volume may shift the baseline of the laser Doppler signal. This limitation means that our findings for basal intramuscular perfusion should be interpreted cautiously, and it may partly explain the contrast with findings in anaesthetised rats [5].

A major advantage of the laser Doppler technique is the possibility to study vasomotion. The origin and control of microvascular vasomotion is still a matter of debate. A central neurogenic regulatory mechanism is suggested by synchronicity on contralateral limbs [29] and by the suppressive effect of central sympathectomy [30]. However, local administration of vasoactive substances such as acetylcholine and sodium nitroprusside directly influences vasomotion [31]. Furthermore, vasomotion has been shown in isolated small arteries, indicating a local regulatory mechanism [32]. In view of these considerations, it can be suggested that vasomotion is regulated by both local vasoactive substances and influences of the central nervous system. The contribution of different regulatory mechanisms can be investigated by analysing the contribution of different frequency intervals to the variability of the laser Doppler signal [16]. In the present study hyperinsulinaemia increased the contribution of the frequencies between 0.01 and $0.04 \mathrm{~Hz}$ in muscle. Stefanovska and co-workers have demonstrated that endothelium-dependent vasodilators specifically increase the contribution of frequencies around $0.01 \mathrm{~Hz}$ and concluded that the frequency interval between 0.01 and $0.02 \mathrm{~Hz}$ represents endothelial activity $[16,31]$. The insulin-induced increase in this frequency interval in muscle in the present study is in accordance with a similar insulin-induced increase in hand skin, found in a previous study [8]. It is also in agreement with present and previous findings of an insulinmediated increase in endothelium-dependent vasodilation in microvasculature [8] and, in general, with insulin-mediated increases in endothelium-dependent vasodilation in resistance vessels [17, 33]. Stefanovska and co-workers have also demonstrated a frequency peak between 0.03 and $0.04 \mathrm{~Hz}$, which is attributed to neurogenic activity, because it has been shown to disappear completely after ganglionic nerve blockade and after sympathectomy [34]. Our results demonstrate an insulin-mediated increase in the contribution of the frequencies around 0.03 and $0.04 \mathrm{~Hz}$. Part of the insulin-mediated effects on microvascular vasomotion might thus be explained by insulin-mediated effects on neurogenic activity. Other studies in larger vessels have demonstrated that hyperinsulinaemia has a stimulatory effect on sympathetic neural activation $[35,36]$. Taken together, our data suggest that an insulin-mediated effect on microvascular vasomotion occurs by increasing endothelial and neurogenic activity.

In the current study we demonstrate consistency in insulin-mediated increases in microvascular reactive hyperaemia in finger skin and leg muscle, and in insulin-mediated increases in microvascular endothelial activity in both finger skin and leg muscle. Although different methods were used to measure microvascular reactive hyperaemia and endothelial function in hand skin and muscle, our results suggest that the effect of insulin on skin and muscle microvasculature takes place simultaneously.

Peak reactive hyperaemia in finger skin is used to detect functional recruitment of initially non-perfused capillaries. Capillaries, especially in the skin, are either intermittently or continuously perfused in the resting state [37, 38]. Only continuously perfused capillaries were counted in the resting state (baseline capillary density), because intermittently perfused capillaries are an important functional reserve that can be recruited after arterial occlusion [37, 38]. Functional capillary recruitment can serve as a mechanism to satisfy increased metabolic demands such as during insulin infusion [8]. Our present and previous data suggest that functional 
capillary recruitment plays a role in insulin sensitivity and blood pressure regulation by modifying both the availability of insulin and glucose to muscle cells, and total peripheral resistance $[8,39,40]$.

In contrast with our previous study [8], but in agreement with previous findings in resistance vessels $[41,42]$, we did not in the present study find any insulin-mediated effect on endothelium-independent vasodilation in finger skin microvasculature. Apparently, other factors not identified in these studies modulate the effect of insulin on endothelium-independent vasodilation in healthy volunteers.

We did not use hand warming to arterialise venous blood samples during the hyperinsulinaemic clamps, because this has been shown to induce unwanted systemic effects on vascular function [43]. Theoretically, the lack of arterialisation of venous blood samples may have led to slightly overestimated insulin sensitivity values [15]. However, to our knowledge, measurements of insulin sensitivity with and without arterialisation have been similar in all previous studies $[43,44,45,46]$. In any case, this issue does not affect our conclusions with regard to insulin's microvascular effects. Finally, we clamped at euglycaemia (i.e. at $5 \mathrm{mmol} / \mathrm{l}$ [18]) instead of at isoglycaemia, which resulted in slightly higher glucose levels during insulin than during saline infusion. It is not clear whether this difference affected our results, but because hyperglycaemia impairs microvascular function [47, 48], we may, if anything, have somewhat underestimated insulin-mediated effects on microvascular function.

In conclusion, we have used intramuscular laser Doppler measurements to measure the effects of insulin on microvascular perfusion in muscle and in skin. Our data show that insulin augments the intramuscular microvascular response to reactive hyperaemia after arterial occlusion. In addition, insulin modulates microvascular blood flow oscillations in the muscle, indicating an increased contribution of endothelial and neurogenic activity. We demonstrate a concomitant effect of insulin on total blood flow and skin microvascular measurements. Hence, this study demonstrates that insulin stimulates not only total blood flow and skin microvascular perfusion, but also microvascular perfusion in human muscle.

Acknowledgements. This work was supported by a grant from The Netherlands Organization for Health Research and Development (ZonMw 940-37-025). We also acknowledge helpful discussions with Professor Michael G. Clark.

\section{References}

1. Baron AD (1994) Hemodynamic actions of insulin. Am J Physiol 267:E187-E202

2. Rattigan S, Clark MG, Barrett EJ (1997) Hemodynamic actions of insulin in rat skeletal muscle: evidence for capillary recruitment. Diabetes 46:1381-1388
3. Rattigan S, Clark MG, Barrett EJ (1999) Acute vasoconstriction-induced insulin resistance in rat muscle in vivo. Diabetes 48:564-569

4. Clark AD, Youd JM, Rattigan S, Barrett EJ, Clark MG (2001) Heterogeneity of laser Doppler flowmetry in perfused muscle indicative of nutritive and nonnutritive flow. Am J Physiol Heart Circ Physiol 280:H1324-H1333

5. Clark AD, Barrett EJ, Rattigan S, Wallis MG, Clark MG (2001) Insulin stimulates laser Doppler signal by rat muscle in vivo, consistent with nutritive flow recruitment. Clin Sci 100:283-290

6. Raitakari M, Knuuti MJ, Ruotsalainen U et al. (1995) Insulin increases blood volume in human skeletal muscle: studies using [15O]CO and positron emission tomography. Am J Physiol 269:E1000-E1005

7. Coggins M, Lindner J, Rattigan S et al. (2001) Physiologic hyperinsulinemia enhances human skeletal muscle perfusion by capillary recruitment. Diabetes 50:2682-2690

8. Serné EH, IJzerman RG, Gans RO et al. (2002) Direct evidence for insulin-induced capillary recruitment in skin of healthy subjects during physiological hyperinsulinemia. Diabetes 51:1515-1522

9. Shamim-Uzzaman QA, Pfenninger D, Kehrer C et al. (2002) Altered cutaneous microvascular responses to reactive hyperaemia in coronary artery disease: a comparative study with conduit vessel responses. Clin Sci 103:267273

10. Vuilleumier P, Decosterd D, Maillard M, Burnier M, Hayoz D (2002) Postischemic forearm skin reactive hyperemia is related to cardovascular risk factors in a healthy female population. J Hypertens 20:1753-1757

11. Kvernebo K, Staxrud LE, Salerud EG (1990) Assessment of human muscle blood perfusion with single-fiber laser Doppler flowmetry. Microvasc Res 39:376-385

12. Hoffmann U, Uckay I, Fischer M, Wen S, Franzeck UK, Bollinger A (1995) Simultaneous assessment of muscle and skin blood fluxes with the laser- Doppler technique. Int J Microcirc Clin Exp 15:53-59

13. Expert Committee on the Diagnosis and Classification of Diabetes Mellitus (1997) Report of the Expert Committee on the Diagnosis and Classification of Diabetes Mellitus. Diabetes Care 20:1183-1197

14. Leahy MJ, Mul FF de, Nilsson GE, Maniewski R (1999) Principles and practice of the laser-Doppler perfusion technique. Technol Health Care 7:143-162

15. Ferrannini E, Mari A (1998) How to measure insulin sensitivity. J Hypertens 16:895-906

16. Stefanovska A, Bracic M, Kvernmo HD (1999) Wavelet analysis of oscillations in the peripheral blood circulation measured by laser Doppler technique. IEEE Trans Biomed Eng 46:1230-1239

17. Cleland SJ, Petrie JR, Ueda S, Elliott HL, Connell JM (1999) Insulin-mediated vasodilation and glucose uptake are functionally linked in humans. Hypertension 33:554558

18. Wong BJ, Wilkins BW, Holowatz LA, Minson CT (2003) Nitric oxide synthase inhibition does not alter the reactive hyperemic response in the cutaneous circulation. J Appl Physiol 95:504-510

19. Eringa EC, Stehouwer CD, Merlijn T, Westerhof N, Sipkema P (2002) Physiological concentrations of insulin induce endothelin-mediated vasoconstriction during inhibition of NOS or PI3-kinase in skeletal muscle arterioles. Cardiovasc Res 56:464-471

20. Cardillo C, Nambi SS, Kilcoyne CM et al. (1999) Insulin stimulates both endothelin and nitric oxide activity in the human forearm. Circulation 100:820-825 
21. Steinberg HO, Brechtel G, Johnson A, Fineberg N, Baron AD (1994) Insulin-mediated skeletal muscle vasodilation is nitric oxide dependent. A novel action of insulin to increase nitric oxide release. J Clin Invest 94:1172-1179

22. Engelke KA, Halliwill JR, Proctor DN, Dietz NM, Joyner MJ (1996) Contribution of nitric oxide and prostaglandins to reactive hyperemia in human forearm. J Appl Physiol $81: 1807-1814$

23. Dakak N, Husain S, Mulcahy D et al. (1998) Contribution of nitric oxide to reactive hyperemia: impact of endothelial dysfunction. Hypertension 32:9-15

24. Meredith IT, Currie KE, Anderson TJ, Roddy MA, Ganz P, Creager MA (1996) Postischemic vasodilation in human forearm is dependent on endothelium-derived nitric oxide. Am J Physiol 270:H1435-H1440

25. Tagawa T, Imaizumi T, Endo T, Shiramoto M, Harasawa Y, Takeshita A (1994) Role of nitric oxide in reactive hyperemia in human forearm vessels. Circulation 90:2285-2290

26. Vollenweider P, Tappy L, Randin D et al. (1993) Differential effects of hyperinsulinemia and carbohydrate metabolism on sympathetic nerve activity and muscle blood flow in humans. J Clin Invest 92:147-154

27. Vincent MA, Dawson D, Clark AD et al. (2002) Skeletal muscle microvascular recruitment by physiological hyperinsulinemia precedes increases in total blood flow. Diabetes 51:42-48

28. Neviere R, Mathieu D, Chagnon JL, Lebleu N, Millien JP, Wattel F (1996) Skeletal muscle microvascular blood flow and oxygen transport in patients with severe sepsis. Am J Respir Crit Care Med 153:191-195

29. Schechner JS, Braverman IM (1992) Synchronous vasomotion in the human cutaneous microvasculature provides evidence for central modulation. Microvasc Res 44:27-32

30. Bernardi L, Rossi M, Fratino P, Finardi G, Mevio E, Orlandi C (1989) Relationship between phasic changes in human skin blood flow and autonomic tone. Microvasc Res 37:16-27

31. Kvandal P, Stefanovska A, Veber M, Desiree KH, Arvid KK (2003) Regulation of human cutaneous circulation evaluated by laser Doppler flowmetry, iontophoresis, and spectral analysis: importance of nitric oxide and prostaglandines. Microvasc Res 65:160-171

32. Davis MJ, Sikes PJ (1990) Myogenic responses of isolated arterioles: test for a rate-sensitive mechanism. Am J Physiol 259:H1890-H1900

33. Mather K, Laakso M, Edelman S, Hook G, Baron A (2000) Evidence for physiological coupling of insulin-mediated glucose metabolism and limb blood flow. Am J Physiol Endocrinol Metab 279:E1264-E1270

34. Kastrup J, Bulow J, Lassen NA (1989) Vasomotion in human skin before and after local heating recorded with laser Doppler flowmetry. A method for induction of vasomotion. Int J Microcirc Clin Exp 8:205-215

35. Anderson EA, Hoffman RP, Balon TW, Sinkey CA, Mark AL (1991) Hyperinsulinemia produces both sympathetic neural activation and vasodilation in normal humans. J Clin Invest 87:2246-2252
36. Rowe JW, Young JB, Minaker KL, Stevens AL, Pallotta J, Landsberg L (1981) Effect of insulin and glucose infusions on sympathetic nervous system activity in normal man. Diabetes 30:219-225

37. Serné EH, Gans RO, Maaten JC ter, Tangelder GJ, Donker AJ, Stehouwer CD (2001) Impaired skin capillary recruitment in essential hypertension is caused by both functional and structural capillary rarefaction. Hypertension 38:238242

38. Antonios TF, Rattray FE, Singer DR, Markandu ND, Mortimer PS, MacGregor GA (1999) Maximization of skin capillaries during intravital video-microscopy in essential hypertension: comparison between venous congestion, reactive hyperaemia and core heat load tests. Clin Sci 97:523-528

39. Serné EH, Stehouwer CD, Maaten JC ter et al. (1999) Microvascular function relates to insulin sensitivity and blood pressure in normal subjects. Circulation 99:896902

40. Serné EH, Gans RO, Maaten JC ter, Wee PM ter, Donker AJ, Stehouwer CD (2001) Capillary recruitment is impaired in essential hypertension and relates to insulin's metabolic and vascular actions. Cardiovasc Res 49:161168

41. Steinberg HO, Chaker H, Leaming R, Johnson A, Brechtel G, Baron AD (1996) Obesity/insulin resistance is associated with endothelial dysfunction. Implications for the syndrome of insulin resistance. J Clin Invest 97:2601-2610

42. Taddei S, Virdis A, Mattei P, Natali A, Ferrannini E, Salvetti A (1995) Effect of insulin on acetylcholineinduced vasodilation in normotensive subjects and patients with essential hypertension. Circulation 92:2911-2918

43. Petrie JR, Ueda S, Morris AD, Elliott HL, Connell JM (1996) Potential confounding effect of hand-warming on the measurement of insulin sensitivity. Clin Sci 91:65-71

44. Wahab PJ, Rijnsburger AW, Oolbekkink M, Heine RJ (1992) Venous versus arterialised venous blood for assessment of blood glucose levels during glucose clamping: comparison in healthy men. Horm Metab Res 24:576579

45. Andrews J, Klimes I, Vasquez B, Nagulesparan M, Reaven GM (1984) Can mixed venous blood be used to measure insulin action during the hyperinsulinemic clamp? Horm Metab Res 16 [Suppl 1]:164-166

46. Nauck MA, Blietz RW, Qualmann C (1996) Comparison of hyperinsulinaemic clamp experiments using venous, 'arterialized' venous or capillary euglycaemia. Clin Physiol 16:589-602

47. Renaudin C, Michoud E, Rapin JR, Lagarde M, Wiernsperger N (1998) Hyperglycaemia modifies the reaction of microvessels to insulin in rat skeletal muscle. Diabetologia 41:26-33

48. Akbari CM, Saouaf R, Barnhill DF, Newman PA, LoGerfo FW, Veves A (1998) Endothelium-dependent vasodilatation is impaired in both microcirculation and macrocirculation during acute hyperglycemia. J Vasc Surg 28:687694 